\title{
Low latency IR-HARQ for multiband WLAN system
}

\author{
Masanori Yofune ${ }^{\text {a) }}$, Satoshi Sonobe, Atsuhiko Sugitani, \\ Yasuharu Amezawa, and Shin-ich Sato \\ Mobile Techno Corp., \\ 4-4-5 Minatomirai, Nishi-ku, Yokohama, Kanagawa 220-0012, Japan \\ a)yofune.masanori@jp.fujitsu.com
}

\begin{abstract}
This paper proposes a low latency incremental redundancy hybrid ARQ (IR-HARQ) scheme for a multiband wireless local area network (WLAN) system. The proposed scheme can reduce a retransmission delay and ensure backward compatibility with WLAN devices by using a clear to send (CTS) frame of a current WLAN system and rate compatible lowdensity parity-check (RC-LDPC) codes. Additionally, it can minimize the retransmission delay by selecting an optimal retransmission data length on the basis of the accumulated mutual information (ACMI) for available frequency bands. The results of computer simulations show that the multiband WLAN system using the proposed scheme has shorter retransmission delay and higher system throughput than the current WLAN system.
\end{abstract}

Keywords: wireless LAN, multiband, IR-HARQ, RC-LDPC, ACMI

Classification: Wireless Communication Technologies

\section{References}

[1] N. Egashira, K. Yano, S. Tsukamoto, J. Webber, M. Sutoh, Y. Amezawa, and T. Kumagai, "Integrated synchronization scheme for WLAN systems employing multiband simultaneous transmission," Proc. IEEE Wireless Communications and Networking Conference (WCNC 2017), San Francisco, USA, Mar. 2017. DOI:10.1109/WCNC.2017.7925799

[2] D. Chase, "Code combining-A maximum likelihood decoding approach for combing and arbitrary number of noisy packets," IEEE Trans. Commun., vol. 33, no. 5, pp. 385-393, May 1985. DOI:10.1109/TCOM.1985.1096314

[3] H. Saber and I. Marsland, "An incremental redundancy hybrid ARQ scheme via puncturing and extending of polar codes," IEEE Trans. Commun., vol. 63, no. 11, pp. 3964-3973, Sept. 2015. DOI:10.1109/TCOMM.2015.2477082

[4] M. Yofune, S. Sonobe, A. Sugitani, Y. Amezawa, and S. Sato, "Low latency IRHARQ scheme for WLAN system," Proc. IEEE 13th Malaysia International Conference on Communications (MICC 2017), Nov. 2017.

[5] IEEE Std. 802.11-2012, "IEEE Standard for Information technology-Telecommunications and information exchange between systems, Local and metropolitan area networks-Specific requirements Part 11: Wireless LAN Medium Access Control (MAC) and Physical Layer (PHY) Specifications," Mar. 2012. DOI:10.1109/IEEESTD.2012.6178212 
[6] J. Chen, A. Dholakia, E. Eleftheriou, P. C. Fossorier, and X. Y. Hu, "Reducedcomplexity decoding of LDPC codes," IEEE Trans. Commun., vol. 53, no. 8, pp. 1288-1299, Aug. 2005. DOI:10.1109/TCOMM.2005.852852

\section{Introduction}

As wireless local area networks (WLANs) have increasingly been used, congestion and shortage of frequency resources regularly occur at 2.4 and $5 \mathrm{GHz}$. To overcome this problem, a multiband WLAN system has recently been proposed that simultaneously transmits data packets of frequency bands in the current IEEE802.11 WLAN system [1]. This system can improve the receiving performance and band occupancy time by using simultaneous transmission data packets that divide the original data into frequency bands and add a media access control (MAC) header. However, the MAC header makes the retransmission overhead in this system larger than that in the current WLAN system. Especially, when the number of retransmissions is increased by a multipath fading channel that causes an uncorrected modulation and coding scheme (MCS) selection, the larger overhead degrades system throughput.

A chase-combining hybrid ARQ (CC-HARQ) and an incremental redundancy hybrid ARQ (IR-HARQ) are generally valid approaches to improve the receiving and decoding performance in retransmission $[2,3]$. In the CC-HARQ scheme, the receiving performance can be improved by combining recently received packets with the same packet received in the past. On the other hand, in the IR-HARQ scheme, the decoding performance improves as the number of retransmissions increases since some redundancy bits are retransmitted by rotation. Therefore, the IR-HARQ scheme has smaller retransmission overhead than the CC-HARQ scheme. However, the current WLAN system does not support the HARQ scheme. For the current WLAN system to support the HARQ scheme, we have proposed a low latency IR-HARQ scheme [4]. This scheme can ensure backward compatibility with WLAN devices by using a control frame of the current WLAN system and rate compatible low-density parity-check (RC-LDPC) codes that are designed on the basis of IEEE802.11n LDPC codes.

To reduce the retransmission delay in the multiband WLAN system, this paper proposes a low latency IR-HARQ scheme for the multiband WLAN system. The proposed scheme can minimize the retransmission delay by selecting an optimal retransmission data length on the basis of the accumulated mutual information (ACMI) for available frequency bands. In addition, it can ensure the above backward compatibility by adopting RC-LDPC codes and reporting the retransmission data length of the IR-HARQ scheme by using a clear to send (CTS) frame. Results of computer simulations show that the multiband WLAN system using the proposed scheme has a lower average transmission delay and higher system throughput than the current WLAN system that adopts IEEE802.11n LDPC codes [5]. 


\section{Proposed low latency IR-HARQ scheme}

This section describes the proposed low latency IR-HARQ protocol and the method for calculating the optimal retransmission data length on the basis of the ACMI for the multiband WLAN system.

\subsection{IR-HARQ protocol for the multiband WLAN system}

Fig. 1 shows the retransmission protocol for the conventional scheme in the current WLAN system and the proposed scheme for the multiband WLAN system. As shown in Fig. 1(a), the conventional scheme must transmit a request to send (RTS) and CTS frames before the transmission of data packets to avoid packet collision. In addition, if the retransmission user does not have the minimum random back-off time during a contention window (CW) after failing to receive a data packet, the retransmission delay increases. On the other hand, as shown in Fig. 1(b), the proposed scheme can give the retransmission packet the highest priority without a packet collision by informing all users about the retransmission by using the CTS/NACK frame. The retransmission procedure of the proposed IR-HARQ scheme is as follows.

Step 1. A source transmits the RTS frame at desired frequency bands.

Step 2. A destination transmits the CTS frame at available frequency bands that received the RTS frame.

Step 3. The source simultaneously transmits the divided data packets at frequency bands that received the CTS frame. In the following steps, all transmission and receiving processes use the same frequency bands.

Step 4. The destination combines the demodulated data sequences for the above divided packets and decodes the combined sequence after storing the packets in a HARQ buffer. If the retransmission packet is received, the destination decodes the data sequence that combines the stored data of the HARQ buffer and the demodulated data for the retransmission packet.

Step 5. The destination decides whether the result of a frame check sequence (FCS) check for received packets contains errors.

Step 6. If the result of the FCS check contains no errors, the destination transmits an acknowledgement (ACK) frame and clears the HARQ buffer.

Step 7. If the result of the FCS check contains errors, the destination transmits the CTS/NACK frame after setting a value of a network allocation vector (NAV) that is calculated from an optimal retransmission data length.

Step 8. If the source receives the ACK frame or a retransmission counter has reached an upper limit, the source finishes the retransmission process after clearing the retransmission counter.

Step 9. If the retransmission counter has not reached the upper limit, the source transmits the retransmission data packets that have the calculated retransmission data length from the NAV value of the CTS/NACK frame. After increasing the retransmission counter by one, return to Step 4 . 


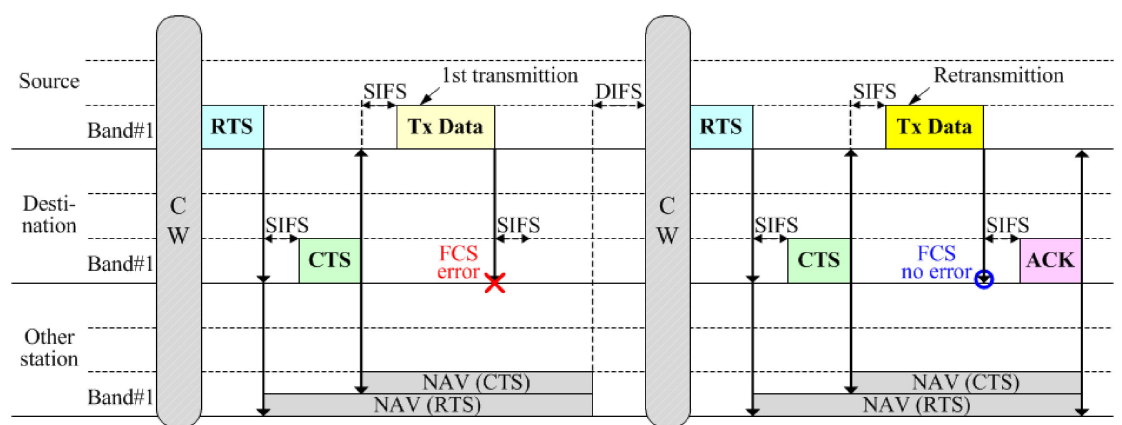

(a) Conventional: retransmission scheme in current WLAN system with RTS/CTS mode

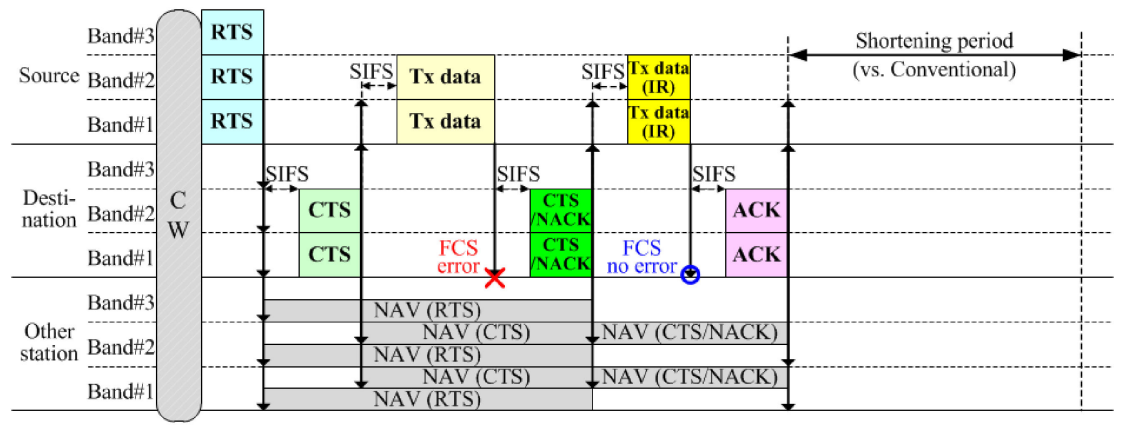

(b) Proposal: IR-HARQ scheme in multiband WLAN system $\begin{array}{lll}\text { RTS : Request to send } & \text { CW : Contention window } & \text { DIFS : Distributed coordination function inter frame space } \\ \text { CTS : Clear to send } & \text { NAV : Network allocation vector } & \text { FCS : Frame check sequence } \\ \text { ACK : Acknowledgement } & \text { SIFS : Short inter frame space } & \text { IR : Incremental redundancy }\end{array}$

Fig. 1. Retransmission protocols: (a) conventional, (b) proposal.

transmission to other stations by the CTS/NACK frame. As a result, the proposed scheme can quickly transmit the retransmission packet without a $\mathrm{CW}$ period and RTS/CTS sequence in the retransmission.

\subsection{Optimization of the retransmission data length}

In the IR-HARQ scheme, to minimize the retransmission delay, the retransmission data length must be minimized in accordance with the channel condition. In the multiband WLAN system, to succeed in decoding the combined sequence in the $n$-th transmission, the amount of ACMI, which is the left term in (1), has to be larger than the target data rate in the first transmission $R_{1}$.

$$
\left(\sum_{m=1}^{n} N_{m} \sum_{f=1}^{F} \bar{C}_{m, f}\right) \div N_{1}>R_{1}
$$

Therefore, if the decoding of the $n$-th transmission fails, the proposed scheme can minimize the retransmission delay by selecting the minimum value for the next transmission data length $N_{n+1}$ that satisfies (2).

$$
\begin{aligned}
& N_{n+1} \sum_{f=1}^{F} \bar{C}_{n+1, f}>N_{1} R_{1}-\sum_{m=1}^{n} N_{m} \sum_{f=1}^{F} \bar{C}_{m, f}, \\
& \bar{C}_{m, f}=\frac{1}{N_{s c, f}} \sum_{k=1}^{N_{s c, f}}\left(L-\int_{v \in \mathbf{C}} \frac{e^{-|v|^{2}}}{\pi 2^{L}} \sum_{x \in \chi} \sum_{i=1}^{L} \log _{2} A_{v, x, i}\left(S N R_{k, f}\right) d v\right),
\end{aligned}
$$

where $N_{m}$ is the number of data symbols for the $2^{L}$-ary modulation scheme $\chi$ of the $m$-th transmission, $N_{s c, f}$ is the number of subcarriers at the $f$-th frequency band, and $\mathbf{C}$ is the complex field. If the bit-interleaved coded modulation (BICM) is used 
in the $m$-th transmission, the capacity $\bar{C}_{m, f}$ is given by (3) and (4). For other coding scheme besides the BICM, the capacity is given by (3) and (5), which approximates (4) by the signal to noise ratio (SNR) offset $\alpha$.

$$
\begin{aligned}
& A_{v, x, i}\left(S N R_{k, f}\right)=1+\left(\sum_{\mathrm{B}_{i}(\hat{x})=1-\mathrm{B}_{i}(x)} e^{-\mid(x-\hat{x}) \sqrt{S N R_{k, f}+\left.\nu\right|^{2}}} / \sum_{\mathrm{B}_{i}(\hat{x})=\mathrm{B}_{i}(x)} e^{-\left|(x-\tilde{x}) \sqrt{S N R_{k, f}}+v\right|^{2}}\right), \\
& A_{v, x, i}\left(S N R_{k, f}\right)=1+\left(\sum_{\mathrm{B}_{i}(\hat{x})=1-\mathrm{B}_{i}(x)} e^{-\left|(x-\hat{x}) \sqrt{S N R_{k, f}+\alpha}+v\right|^{2}} / \sum_{\mathrm{B}_{i}(\hat{x})=\mathrm{B}_{i}(x)} e^{-\left|(x-\tilde{x}) \sqrt{S N R_{k, f}+\alpha}+\nu\right|^{2}}\right),
\end{aligned}
$$

where $B_{i}(x)$ is the $i$-th bit of the symbol $x$ and $S N R_{k, f}$ is the estimated SNR in the $k$-th subcarrier at the $f$-th frequency band to accommodate frequency selective fading.

\section{Performance evaluation}

This section evaluates the average transmission delay and the system throughput for the conventional and proposed schemes. In this letter, the conventional scheme (Conventional) is the retranmission scheme in Fig. 1(a) with IEEE802.11n LDPC codes, in which the coding rate is supported from 0.5 to 0.833 . In contrast, the proposed scheme (Proposal) is the retranmission scheme in Fig. 1(b) with designed RC-LDPC codes [4], in which the coding rate is supported from 0.5 to 0.875 . In addition, Conventional and Proposal calculate the retransmission data length by (4) and (5) with $\alpha=1.0 \sim 1.4$ [4], respectively. This evaluation adopts a layered belief propagation (LBP) decoding [6] that uses 15 iterations; QPSK, 16QAM, and 64QAM modulations; the IEEE802.11a standard frame format with $20 \mathrm{MHz}$ bandwidth; the packet payload of 157 882 bytes; and a 6-path exponential decay model with $2 \mathrm{~dB}$ decay. The maximum number of retransmission is four.

Fig. 2 shows the transmitter and receiver (TRX) block diagram for the multiband WLAN system with the proposed IR-HARQ scheme. In this system, the demodulated data sequence for received data packets is decoded after the packets are combined and stored in the HARQ combiner. After the transmission of the CTS/NACK or ACK frame is requested from the HARQ controller at the destination in accordance with the result of the FCS check, the CTS/NACK or ACK frame is transmitted to the source. If the source receives the CTS/NACK frame, after the HARQ controller in the source reports the optimal retransmission data length that is calculated from the NAV value of the CTS/NACK frame to the encoder in the source, the additional parity bits are transmitted to the destination.

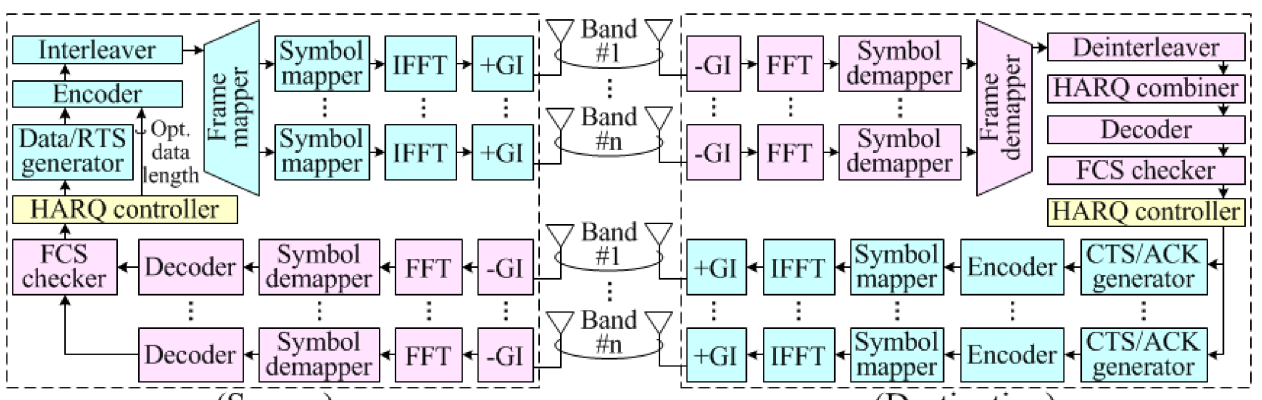

(Source)

(Destination)

Fig. 2. TRX block diagram for multiband WLAN system with proposed IR-HARQ scheme. 


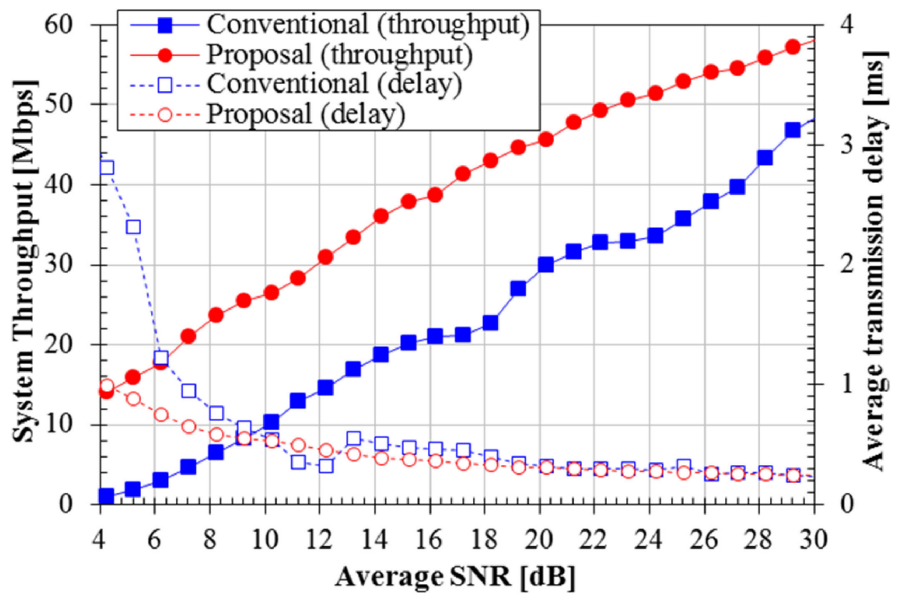

Fig. 3. System throughputs and average transmission delay for Conventional and Proposal with AMC.

Fig. 3 shows the average transmission delays and system throughputs for Conventional and Proposal with an adaptive modulation and coding scheme (AMC) at 2.4 and $5.7 \mathrm{GHz}$. In this figure, the X-axis is the average SNRs at 5.7 and $2.4 \mathrm{GHz}$ bands. Additionally, Y-axis is the average transmission delay, i.e., the average time from when a packet is generated until it is successfully received or the retransmission limit is reached. As shown in Fig. 3, Proposal reduces the average transmission delay by up to $33 \%$ and increases the system throughput by $150 \%$ on average compared with Conventional. The reason for the improved system throughput at the average $\mathrm{SNR}<10 \mathrm{~dB}$ is the improved average transmission delay. On the other hand, the reason for the improved system throughput at the average SNR $>10 \mathrm{~dB}$ is the required SNR that improved by the IR-HARQ scheme.

\section{Conclusion}

This paper proposed a low latency incremental redundancy hybrid ARQ (IRHARQ) scheme for a multiband wireless local area network (WLAN) system and evaluated the average transmission delays and system throughputs for the proposed scheme and the current WLAN system. The results of computer simulations showed the multiband WLAN system using the proposed scheme has up to $33 \%$ lower average transmission delay and on average $150 \%$ higher system throughput than the current WLAN system.

\section{Acknowledgments}

This work is supported by the Ministry of Internal Affairs and Communications under a grant entitled "Research and Development of Spectral-Efficiency Improvement Technology Employing Simultaneous Transmission over Multiple LicenseExempt Bands.” 